Bistua:Revista de la Facultad de Ciencias Básicas.2016.14(2):43-51

\title{
ASPECTO ESTRUCTURAL MEDIANTE MICROSCOPIA ELECTRONICA DE BARRIDO (SEM) DE ANTOCIANINAS OBTENIDAS MEDIANTE ATOMIZACIÒN
}

\author{
Irina Herazo Camaño ${ }^{1}$, Guillermo Arrázola Paternina1, Enrique Pardo Perez² \\ 1 Universidad de Córdoba, Facultad de Ingenierías, Programa de Ingeniería de Alimentos. Montería, \\ Colombia. \\ ¿Universidad de Córdoba, Facultad de Ciencias Básicas, Departamento de Biología. Montería, \\ Colombia.
}

\section{Resumen}

Este artículo presenta el proceso de extracción de antocianinas de cascara berenjena, con etanol acidificado, temperaturas y tiempos de extracción. Los extractos de antocianinas se micro encapsularon evaluando la influencia de las condiciones de secado por aspersión en las propiedades fisicoquímicas de los extractos. Las variables fueron temperatura del aire de entrada $\left(160^{\circ} \mathrm{C}\right.$ y $\left.190^{\circ} \mathrm{C}\right)$ y la concentración de maltodextrina (25\%, 30\% y 35\%). Se realizó análisis de contenido de antocianinas, mediante espectrofotometría electrónica de barrido. La más alta concentración de antocianinas en cascara de berenjena $(115 \mathrm{mg} / 100 \mathrm{~g})$ se obtuvo con $53 \%$ etanol, 3 horas y a $29{ }^{\circ} \mathrm{C}$. Durante la microencapsulación por secado en spray, se evaluó estadísticamente que la temperatura y el porcentaje de maltodextrina empleado en la microencapsulación influenció en la mayoría de las propiedades fisicoquímicas del extracto obtenido.

Palabras Clave: Berenjena, Antocianinas, Microencapsulación, Temperatura, Maltodextrina

\section{APPEARANCE BY STRUCTURAL SCANNING ELECTRON MICROSCOPY (SEM) BY ANTHOCYANUNS DERIVED ATOMIZATION}

\section{Abstract}

This article presents the optimization process of extraction of anthocyanins peel eggplant was performed concentrations of acidified ethanol extraction temperatures and times. Anthocyanins extracts micro encapsulated evaluating the influence of spray drying conditions on the physicochemical properties of powders. The variables 
44

were inlet air temperature $\left(160\right.$ to $\left.190^{\circ} \mathrm{C}\right)$ and the concentration of maltodextrin $(25$, 30 and $35 \%$ ). analysis of anthocyanins, color parameters, antioxidant capacity, humidity, water activity, hygroscopicity, solubility, density, and electron spectroscopy scanning were performed. The highest concentration of anthocyanins in peel eggplant $\left(115 \mathrm{mg} / 100 \mathrm{~g}\right.$ ) was obtained with $53 \%$ ethanol, 3 hours and $29{ }^{\circ} \mathrm{C}$. During spray drying microencapsulation, it is statistically observed that the temperature and the percentage of maltodextrin used in the microencapsulation influenced most physicochemical properties of powders.

Keywords: Eggplant, Anthocyanins, Microencapsulation, Temperature, Maltodextrin

*Para citar este artículo: Herazo Camaño I,Arrázola Paternina G, Pardo Perez E.Aspecto estructural mediante microscopia electrónica de barrido (SEM) de antocianinas obtenidas mediante atomización. Revista Bistua. 2016.14(2):43-51

+ Autor para el envió de correspondencia y la solicitud de las separatas Enrique Pardo Perez. Universidad de Córdoba, Facultad de Ciencias Básicas, Departamento de Biología. Montería, Colombia.e-mail:_epardop@correo.unicordoba.edu.co 


\section{Introducción}

El interés por los pigmentos antocianos en investigaciones científicas se han incrementado en los últimos años, debido no sólo al color que confieren a los productos que las contienen sino a su probable papel en la reducción de las enfermedades coronarias, cáncer, diabetes, efectos antiinflamatorios, mejoramiento de la agudeza visual y comportamiento cognitivo; estos efectos terapéuticos positivos, están principalmente asociados con sus propiedades antioxidantes $(1,2)$. Por tanto, además de su papel funcional, las antocianinas como pigmentos naturales se constituyen en agentes potenciales en la obtención de productos con valor agregado para el consumo humano.

En los últimos años, las tendencias mundiales indican un interés acentuado de los consumidores por reemplazar el uso de colorantes artificiales por colorantes de origen natural, que además de ser atractivos para los consumidores aporten beneficios a las funciones biológicas del organismo humano y sean estables en el tiempo(3,4). Sin embargo la mayoría de los pigmentos de origen natural tienen como limitante su baja estabilidad al sufrir diversas transformaciones en el tiempo(4), razón por la que muchas investigaciones se orientan no solo a la obtención de pigmentos naturales sino también a la búsqueda de alternativas de solución que aumenten su estabilidad. Los pigmentos antocianos tienen baja estabilidad debido a la sensibilidad a los cambios de $\mathrm{pH}$, temperatura, luz, oxigeno, entre otros factores, los cuales se convierten en la principal limitación de estos pigmentos para ser aplicados como sustitutos potenciales de los colorantes artificiales en alimentos $(5,6,7,8)$. En la industria las aplicaciones de técnicas de microencapsulación han ido incrementándose debido a la protección de los materiales encapsulados frente a diferentes factores que afectan su vida útil, permitiendo mantener su estabilidad y viabilidad en el tiempo, garantizando su contenido nutricional y su aporte a la salud(4,6). Otros autores han trabajado con diferentes técnicas de microencapsulación de antocianinas con la finalidad de ofrecer la protección y estabilidad de estos pigmentos, mejorando de esta forma su utilización como ingredientes alimenticios $(4,6,9,10,11,12)$.

objetivo de esta investigación fue obtener y microencapsular las antocianinas de cáscara de berenjena mediante la microencapsulación molecular con $\beta$-ciclodextrina $y$ verificación de la morfología de las microcápsulas a concentraciones del agente encapsulante.

Materiales y Métodos

Microscopia electrónica de Barrido (SEM) de polvos secos en Spray. 
46

La morfología de las partículas se evaluó por microscopía electrónica de barrido (SEM), en la Universidad de Antioquia. Los polvos fueron unidos a una cinta adhesiva de doble cara montado sobre talones de SEM, recubierto con 3-5 mA oro / paladio al vacío y se examinaron con un microscopio electrónico de barrido JEOL ${ }^{\circledR}$ modelo JSM 6490 LV, operado en el modo de alto vacío.

Microencapsulación antocianinas por inclusión molecular con ciclodextrina. Para el proceso de microencapsulación se empleó el extracto de antocianinas, previamente concentrado en un rotaevaporador V700 (Büchi $\left.{ }^{\circledR}\right)$ equipado de Bomba de vacío V-700 con controlador $\mathrm{V}-850$, la temperatura de concentración fue de $35 \pm 2^{\circ}$ C La solución concentrada de antocianinas fue almacenada a $4 \stackrel{\circ}{\circ} \mathrm{C}$ en frascos de color ámbar.

Los extractos del pigmento concentrado fueron

microencapsulados utilizando la técnica de inclusión molecular con $\beta$ ciclodextrina $(\beta-C D)$, a través del método de Co- precipitación(13). A través de diversos ensayos preliminares se establecieron relaciones estequiometrias o razones molares de $\beta-C D$ : extracto de antocianina desde 1:5 hasta 1:50 $(13,14)$. La mezcla de antocianinas con la $\beta$-ciclodextrina se realizó en erlenmeyer protegidos de la luz, los cuales se sometieron a agitación con la ayuda de un agitador magnético durante 7 horas a $35^{\circ} \mathrm{C}(12,15)$. Culminado este periodo la mezcla se incubó a $4{ }^{\circ} \mathrm{C}$ por $24 \mathrm{~h}$, para que la reacción alcanzara el equilibrio(16). Finalmente se filtró y el complejo precipitado se sometió a un proceso de liofilizado empleando un equipo liofilizador Labconco modelo

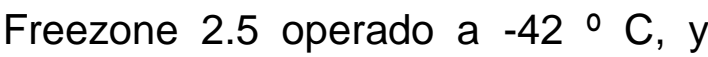
0,013 mBar durante un periodo de 24 horas $(13,17,18)$.Una vez obtenido el extracto microencapsulado y liofilizado, se procedió a determinar el contenido de antocianinas retenido en complejo de inclusión. Para este fin se tomaron $50 \mathrm{mg}$ de polvo y se diluyeron con $30 \mathrm{~mL}$ de agua, se sometió a agitación y se determinó el contenido total de antocianinas monoméricas utilizando el método de $\mathrm{pH}$ diferencial.

La eficiencia del proceso se determinó empleando la siguiente ecuación:

$\%$ de Inclusión $=\left[\mathrm{CA} \times \mathrm{FD}\left(\frac{\mathrm{CM}}{\mathrm{MA}}\right)\right] / \mathrm{CI}$ CA: Contenido de antocianinas en el liofilizado $(\mathrm{g} / \mathrm{ml})$; FD: Factor de dilución; CM: cantidad muestra liofilizada (g); MA: gramos de muestra liofilizada analizada; $\mathrm{Cl}$ : Contenido de antocianinas en el extracto.

Resultados y Discusión. 
47

Microscopia electrónica de Barrido (SEM) de polvos. En la Figura 1, se puede observar la morfología de las microcápsulas a concentraciones de agente encapsulante de $30 \%$ con temperatura de 160 y $190{ }^{\circ} \mathrm{C}$. Las microcápsulas son de tamaño y forma variable, es decir hay capsulas de forma esférica con una superficie lisa y capsulas con superficie abollada o irregular. En la Figura $1 \mathrm{~A}$ y $1 \mathrm{~B}$, se pueden observar que hay mayor cantidad de microcápsulas redondas $y$ de superficie lisa al emplear la temperatura de aire de secado de $190^{\circ} \mathrm{C}$ que a la temperatura de 160 ${ }^{\circ} \mathrm{C}$.

Esta estructura lisa y esférica de microencapsulados con maltodextrina ha sido observada por autores como $(3,9,11,19)$ en sus estudios de microencapsulación por secado aspersión empleando maltodextrinas en extractos de Acay, luteínaenocianina, mango y pigmentos zanahoria negra (Daucuscarota L) respectivamente. Asimismo reportaron partículas que exhibían superficie lisa, $y$ otras que presentaban superficies porosas con abolladuras superficiales en la encapsulación con maltodextrina de pigmentos de antocianos de Mora de Castilla (Rubus glaucus) y tamarillo (Solanum betaceum). La formación de abolladuras en la superficie de la capsula, generalmente puede ser atribuida a la contracción de las partículas durante el secado, debido a la drástica pérdida de humedad seguido de enfriamiento $(3,20,21)$. Por su parte(22) encontró que los equivalentes de dextrosa (DE) de la maltodextrina empleada también influye en la presencia de grietas y hendiduras en la superficie de la capsula, siendo la maltodextrina de bajos $D E$ la que presenta mayor irregularidades y grietas superficiales. Las Figura $2 \mathrm{~A}$ y $2 \mathrm{~B}$, muestran la morfología de las microcápsulas obtenidas con $20 \%$ de maltodextrina, en estas se observan unas microcápsulas apiladas y unidas entre sí. Esto podría ser debido a que la menor concentración de maltodextrina produjo una mayor hidratación de los polvos. ${ }^{23}$ observó un comportamiento similar en la morfología de microcápsulas obtenidas con $8 \%$ de maltodextrina.(10) en su estudio de microencapsulado de Acai (Euterpe oleraceae Mart.) con maltodextrina, pone manifiesto que cuando la temperatura del aire de entrada fue baja, la mayoría de las partículas mostraron una superficie arrugada, y cuando aumentó la temperatura de secado resultó en un mayor número de partículas con superficie lisa. Esto está relacionado con las diferencias en la velocidad de secado, que es más alta para las temperaturas elevadas, causando una evaporación 
48

más rápida del agua y que conduce a la formación de una costra lisa y dura. Las partículas con superficies rugosas pueden tener problemas en sus propiedades de flujo, además sus grandes áreas de contacto pueden hacerlas más susceptibles a reacciones de degradación, tales como la oxidación(20).

\section{Conclusiones}

Tiempos prolongados de extracción del pigmento y temperaturas altas, causan una disminución del contenido de antocianinas en los extractos, lo que demuestra su alta sensibilidad a los tratamientos de extracción severos. Los pigmentos microencapsulados mediante el secado por aspersión con $30 \%$ de maltodextrina y $190{ }^{\circ} \mathrm{C}$ presentaron buenas propiedades fisicoquímicas, con menores contenidos de humedad $(3,43 \%)$ y actividad de agua $(0,26)$ y mayores porcentajes de solubilidad (93.61\%). La morfología de los polvos visualizados a través de la técnica de microscopia electrónica de barrido, permitió verificar la formación de microcápsulas de maltodextrina con antocianinas, las cuales fueron de forma esférica con superficie lisa y con superficie abollada e irregular.

\section{Referencias bibliográficas}

1. Longo L, Vasapollo G. Extraction and identification of anthocyanins from Smilax aspera L. berries.
Food Chemistry. 2006;94:226231.

2. Garzón G. Las antocianinas como colorantes naturales $y$ compuestos bioactivos: revisión. Acta Biológica Colombiana. 2008;13(3):27-36.

3. Escalona S. Encapsulados de luteina-enocianina y su aplicación en alimentos. [Tesis de Maestría]. Santiago de Chile. Universidad de Chile. 2004.

4. Ribeiro F, Stringheta P. Microencapsulamento

antocianinas. Biotecnologia Ciência e Desenvolvimento. 2006;36:18-24.

5. Castañeda A, Pacheco M, Páez M, Rodríguez J, Galán C. Chemical studies of anthocyanins:

A review. Journal Food Chemistry. 2009;113:859-871.

6. Olaya C, Castaño M, Garzón G. Stability of anthocyanins from Rubus glaucus Benth and Solanum betaceum Cav.dark-red strain as affected by temperature, storage time and water activity. Acta Biológica Colombiana. 2009;14(3):141-156.

7. Owusu A. Chemistry Postharvest. En: Introduction to Food Chemistry. Primera Edición. Editorial CRC Press. United States of America. P. 219. 2005.

8. Poo B. Concentración de Antocianinas en Jugo de Cranberries (Vaccinium macrocarpon Ait.) mediante Nanofiltración. [Tesis de Licenciado en Ciencias de los 
49

Alimentos]. Valdivia. Universidad Austral de Chile. 2005.

9. Tonon $\mathrm{R}$, Brabet $\mathrm{C}$, Hubinger $\mathrm{M}$. Anthocyanin stability and antioxidant activity of spray-dried açai (Euterpe oleracea Mart.) juice produced with different carrier agents. Food Research International. 2010;43(3):907-914.

10. Valduga E, Lima L, Prado R, Ferreira F, Treichel H. Extração, secagem por atomização e microencapsulamento de antocianinas do bagaço da uva isabel (Vitis labrusca). Ciênc. Agrotec. 2008;32(5):1568-1574.

11. Ersus S, Yurdagel U. Microencapsulation of anthocyanin pigments of black carrot (Daucuscarota L.) by spray drier. J Food Eng. 2007;80:805812.

12. Provenzi, G., Falcão, L. y Fett, R. 2006. Estabilidade de Antocianinas de Uvas Cabernet Sauvignon com $\beta$ - e $\gamma$ Ciclodextrinas. Braz. J. Food Technol. 9 (3): 165-170.

13. Del Valle E. Cyclodextrins and their uses: a review. Process Biochemistry. 2004;39:10331046.

14. He Y, Fu P, Shen Z, Gao H. Cyclodextrin-based aggregates and characterization by microscopy. Micron. 2008;39:495516.

15. Chen X, Chen R, Guo Z, Li C, Li $P$. The preparation and stability of the inclusion complex of astaxanthin with b-cyclodextrin Food Chemistry. 2007;101:15801584.

16. Zaibunnisa A, Siti $R$, Nur A. Stabilisation of curcumin with $\mathrm{Y}$ cyclodextrin: phase solubility study and its characterization. IPCBEE 7: 9-13. 2011.

17. Kalogeropoulos N, Yannakopoulou K, Gioxari A, Chiou A, Makris D. Polyphenol characterization and encapsulation in b-cyclodextrin of a flavonoid-rich Hypericum perforatum (St John's wort) extract. LWT - Food Science and Technology. 2010;43:882-889

18. Yuan C, Jin Z, Xu X, Zhuang H, Shen $\mathrm{W}$. Preparation and stability of the inclusion complex of astaxanthin with hydroxypropyl-bcyclodextrin. Food Chemistry. 2008;109:264-268.

19. Caparino O, Tang J, Nindo C, Sablani S, Powers J, Fellman J. Effect of drying methods on the physical properties and microstructures of mango (Philippine 'Carabao' var.) powder. Journal of Food Engineering. 2012;111:135-148.

20. Silva PI, Stringheta PC, Teófilo $\mathrm{RF}$, de Oliveira IRN. Parameter optimization for spray-drying microencapsulation of jaboticaba (Myrciaria jaboticaba) peel extracts using simultaneous analysis of responses. Journal of Food Engineering. 2013;117:538554. 
50

21. Saénz C, Tapia S, Chávez J, Robert P. Microencapsulation by spray drying of bioactive compounds from cactus pear (Opuntia ficus-indica). Food Chemistry. 2009;114: 616-622.

22. Cai Y, Corke H. Production and Properties of Spray-dried Amaranthus Betacyanin Pigments. Journal Of Food Science. 2000;65(6):1248-1252.

23. Fazaeli, M., Emam-Djomeh Z, Kalbasi A, Omid M. Effect of spray drying conditions and feed composition on the physical properties of black mulberry juice powder. Food and Bioproducts Processing. 2012;90:667-675

Figura 1A. Fotografía de Microscopía Electrónica de Barrido (SEM) de microcápsulas $30 \% \mathrm{MD}$ y $160{ }^{\circ} \mathrm{C}, 1000 \mathrm{x}$. Fuente: Autor

Figura 1B. Fotografía de Microscopía Electrónica de Barrido (SEM) de microcápsulas $30 \%$ MD y $190{ }^{\circ} \mathrm{C} 5000 x$. Fuente: Autor.

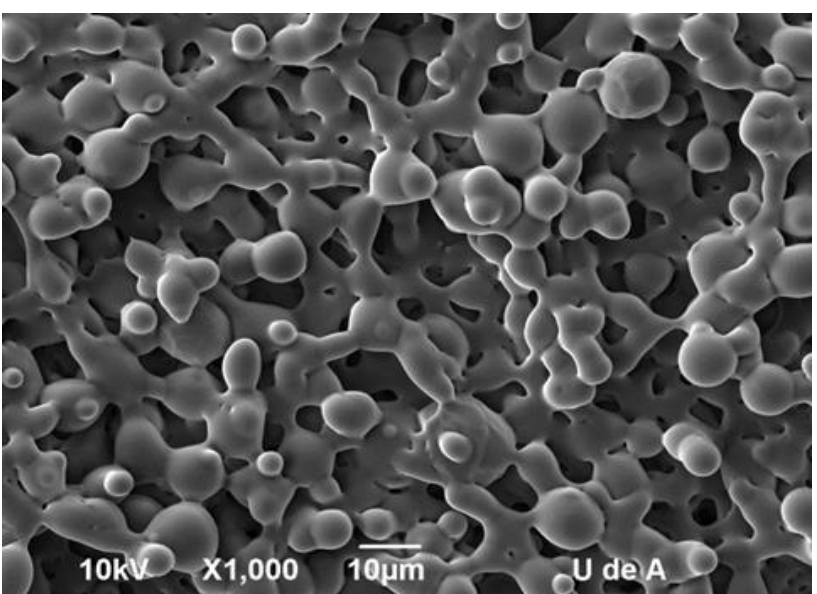

Figura 2A. Fotografías de Microscopía Electrónica de Barrido (SEM) de microcápsulas con $20 \%$ MD y $180{ }^{\circ} \mathrm{C}$, 1000x). Fuente: Autor

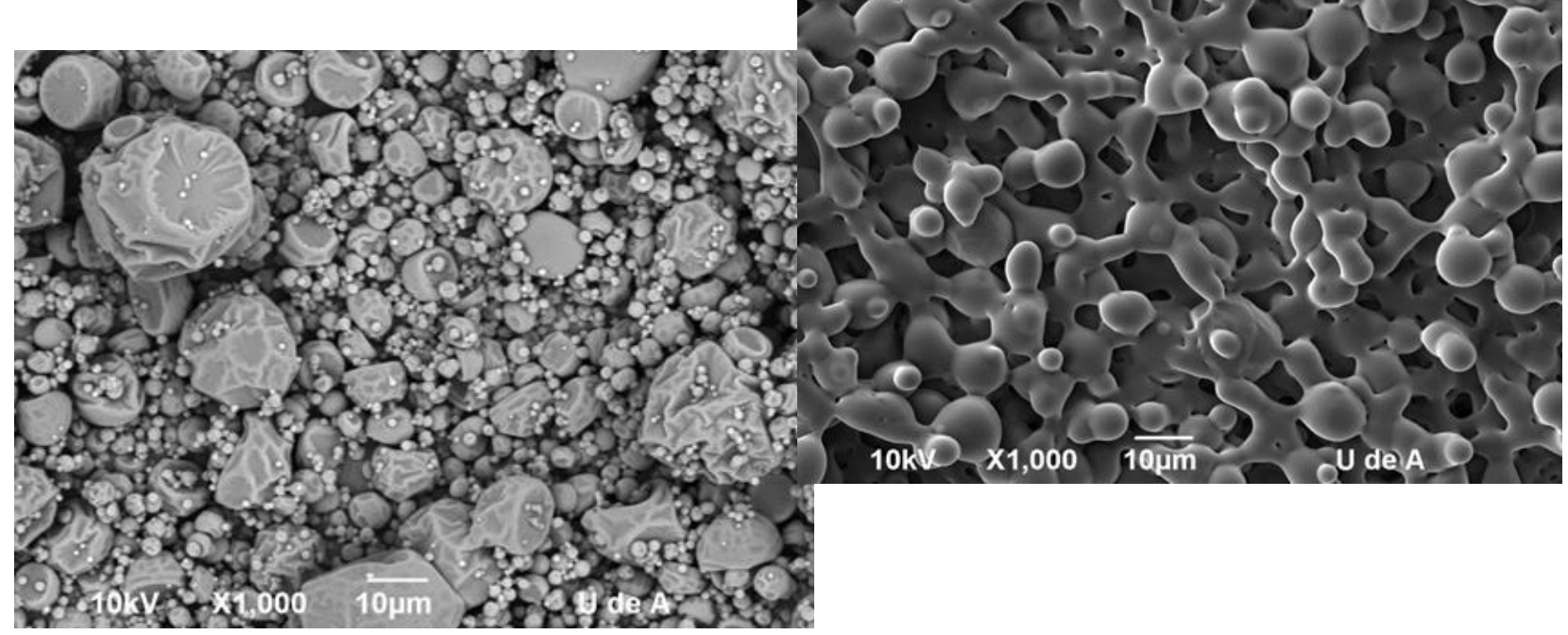


51

Figura 2B. Fotografías de Microscopía Electrónica de Barrido (SEM) de microcápsulas con $20 \% \mathrm{MD}$ y $170{ }^{\circ} \mathrm{C}, 1000 \mathrm{x}$. Fuente: Autor

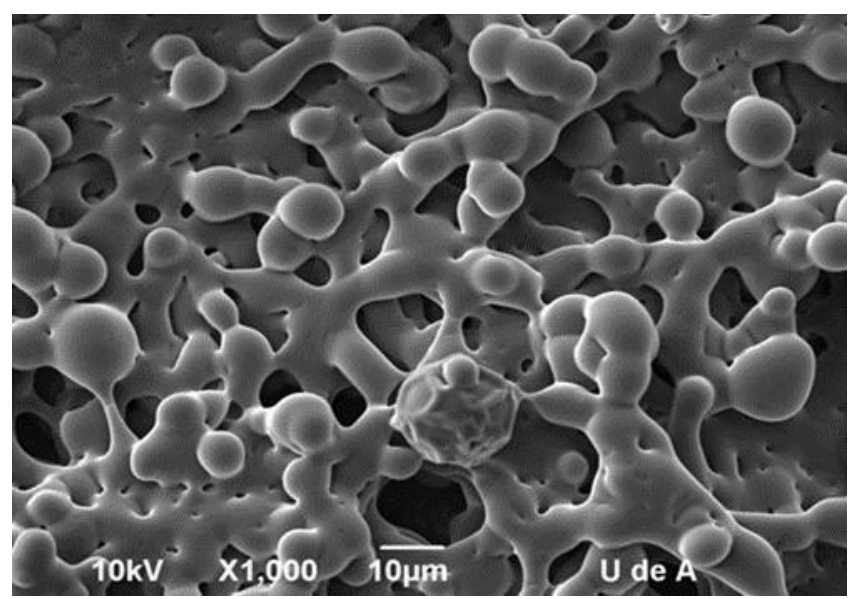

